TRANSACTIONS OF THE

AMERICAN MATHEMATICAL SOCIETY

Volume 281. Number 2, February 1984

\title{
INVARIANT DENSITIES FOR RANDOM MAPS \\ OF THE INTERVAL
}

\author{
BY
}

S. PELIKAN ${ }^{1}$

\begin{abstract}
A random map is a discrete time process in which one of a number of functions is selected at random and applied. Here we study random maps of $[0,1]$ which represent dynamical systems on the square $[0,1] \times[0,1]$. Sufficient conditions for a random map to have an absolutely continuous invariant measure are given, and the number of ergodic components of a random map is discussed.
\end{abstract}

1. Introduction. In this paper we investigate the following kind of question: consider two maps from $[0,1]$ to itself-for example, let $T_{1}(x)=x / 2$ and $T_{2}(x)=$ $2 x(\bmod 1)$. Select an initial point $x_{0} \in[0,1]$, and choose one of the maps at random ( $T_{1}$ with probability $p_{1}$ and $T_{2}$ with probability $p_{2}$ ). Define $x_{1}=T_{i}\left(x_{0}\right)$ with probability $p_{i}, i=1,2$. Continue in this manner, selecting maps at random and setting $x_{n+1}=T_{i}\left(x_{n}\right)$ (prob. $p_{i}$ ). How is the "trajectory" $\left\{x_{n}\right\}$ distributed? We say that a Borel probability measure $\mu$ describes the distribution of $\left\{x_{n}\right\}$ if

$$
\lim _{n \rightarrow \infty} \frac{1}{n} \operatorname{card}\left(\left\{x_{0}, \ldots, x_{n-1}\right\} \cap A\right)=\mu(A)
$$

for every open set $A$ with $\mu(\partial A)=0$. In the specific example mentioned above we prove that when $p_{2}>1 / 2$ there is a unique measure, which is absolutely continuous with respect to Lebesgue measure $m$ and which describes the distribution of the sequence $\left\{x_{n}\right\}$ for $m$-almost every choice of initial condition $x_{0}$ and "almost every" selection of the maps $T_{i}$.

More generally, let $T_{1}(x), \ldots, T_{n}(x)$ be maps of the unit interval and define a "random map" $T$ by $T(x)=T_{i}(x)$ with probability $p_{i}$. A measure $\mu$ will be called $T$-invariant if $\mu(A)=\sum_{i=1}^{n} p_{i} \mu\left(T_{i}^{-1} A\right)$ for each measurable set $A$. We study a class of random maps for which it is possible to describe the distribution of almost every trajectory. This description is accomplished through the demonstration of three facts. The first fact is that every random map may be realized as a transformation of the square $[0,1] \times[0,1]$ to itself. This construction, the pseudo-skew product (see $\S 2$ ), makes the phrase "almost every selection of the maps $T_{i}$ " precise.

The second component of the description is Proposition 6 ( $\$ 5)$ which shows that sets which are invariant for a pseudo-skew product map are products. The third fact

Received by the editors January 27, 1983.

1980 Mathematics Subject Classification. Primary 58F13, 28D99; Secondary 60G10.

'Research sponsored by the Air Force Office of Scientific Research, Air Force Systems Command, USAF, under grant AFOSR-81-0217A. The United States Government is authorized to reproduce and distribute reprints for Government purposes notwithstanding any copyright notation thereon. 
is contained in Theorem 1, which gives sufficient conditions for a random map to have an invariant measure which is absolutely continuous with respect to Lebesgue measure. Such a measure has a finite number of ergodic components $(\S 5)$; taken together these components describe the distribution of almost every trajectory of the random map $T$.

I would like to express my thanks to Professor J. A. Yorke for suggesting this problem and for his continuing interest in this paper.

\section{Background.}

(A) Definitions. A random map of $[0,1]$ is a process $T(x)$ specified by a finite collection of measurable functions $T_{i}:[0,1] \times[0,1], i=1, \ldots, n$, and a probability vector $P=\left(P_{1}, \ldots, P_{n}\right)$. That is, $P_{i} \geqslant 0$ and $\sum_{i=1}^{n} P_{i}=1$. We define $T(x)=T_{i}(x)$ with probability $P_{i}$, and assume that the selection of the functions is an independent identically distributed process so that $T^{m}(x)=T_{i_{m}} \circ T_{i_{m-1}} \circ \cdots \circ T_{i_{1}}(x)$ with probability $\prod_{j=1}^{m} P_{i_{j}}$. A measure $\mu$ on $[0,1]$ is defined to be $T$-invariant if $\mu(A)=$ $\sum_{i=1}^{n} P_{i} \mu\left(T_{i}^{-1}(A)\right)$ for each measurable set $A$.

(B) Pseudo-skew products. Random maps of $[0,1]$ may always be realized as transformations of a larger space. Suppose that $T=T_{i}$ (prob. $\left.P_{i}\right), i=1, \ldots, n$, is a random map. Let $\Omega=\left\{w=\left(w_{i}\right)_{i=0}^{\infty}: w_{i} \in\{1,2, \ldots, n\}\right\}$ be the set of all infinite one-sided sequences of the symbols in $S=\{1,2, \ldots, n\}$. The left shift $\sigma: \Omega \rightarrow \Omega$ is defined by $\left(\sigma\left(w_{i}\right)\right)_{j}=w_{j+1}, j=0,1,2, \ldots$ The topology on $\Omega$ is the product of the discrete topology on $S$, and the Borel measure $\mu_{p}$ on $\Omega$ is defined as the product of the distribution on $S$ given by $\operatorname{Prob}(j)=P_{j}$. Note that there is a 1-1 correspondence between the ways in which the maps $\ldots T_{i_{n}}, T_{i_{n-1}}, \ldots, T_{i_{1}}$ can be selected and points in $\Omega$. The phrase "for almost every selection of the maps $T_{i}$ " means for selections of the maps $T_{i}$ which corresponds to a subset of $\Omega$ having $\mu_{p}$ measure one.

In the space $\Omega \times I$ define a map $R$ by $R(w, x)=\left(\sigma w, T_{w_{0}} x\right)$. The measure $\nu$ is $T$ invariant if and only if $\mu_{p} \times \nu$ is $R$ invariant. We say the random map $T$ is ergodic wrt $\nu$ when $R$ is ergodic wrt $\mu_{p} \times \nu$.

Because there may be no measure on $[0,1]$ which is preserved by each of the $T_{i}$, the map $R$ is not a skew product in the traditional sense [AR]. We will refer to $R$ as the pseudo-skew product (PSP) determined by the random map $T$.

To realize the random map $T$ as a map $S$ of $[0,1] \times[0,1]$ to itself we use the following measure theoretic isomorphism: $\left(\Omega, \sigma, \mu_{p}\right) \approx\left([0,1], m, f_{p}\right)$, where $f$ is defined as follows. Let $I_{1}, \ldots, I_{n}$ be a partition of $[0,1]$ into intervals of length $P_{1}, \ldots, P_{n}$, so that $I_{1}=\left[0, P_{1}\right]$, and $I_{j}=\left[\Sigma^{j-1} P_{i}, \Sigma^{j} P_{i}\right]$. For $x \in I_{j}^{\circ}$ define (Figure 1)

$$
f_{p}(x)=\frac{1}{P_{j}} x-\frac{\Sigma^{j-1} P_{i}}{P_{j}} .
$$

The random map $T$ is then to be represented by $S(x, y)=\left(T_{i}(x), f_{p}(y)\right)$ for $y \in I_{i}$. Note that $T$ has an absolutely continuous invariant measure $\nu \ll m$ if and only if $S$ : $[0,1] \times[0,1] \supset$ has an invariant measure $\nu \times m$ which is absolutely continuous wrt 2-dimensional Lebesgue measure. 


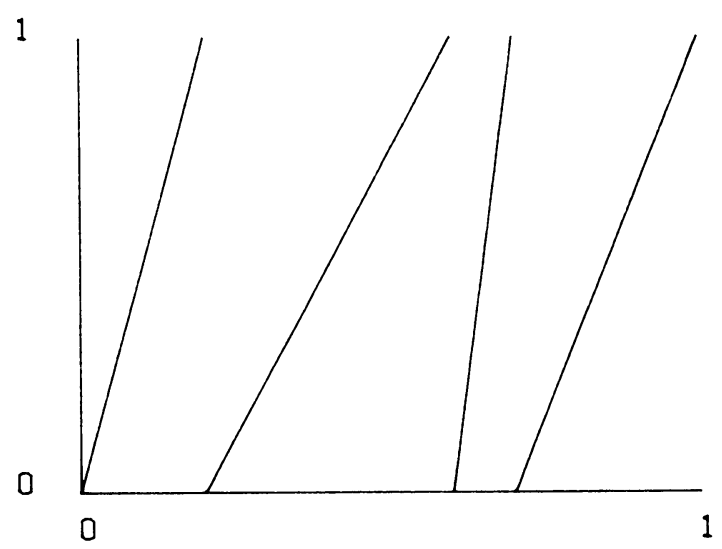

Figure 1. The graph of $f_{p}$

(C) Lasota-Yorke maps and the Frobenius-Perron operator. A transformation $T$ : $[0,1] \rightarrow[0,1]$ is called nonsingular (wrt Lebesgue measure) if $m\left(T^{-1} A\right)=0$ whenever $m(A)=0$. If $T$ is a nonsingular transformation, the action of $T^{*}$ on measures $\left(T^{*} \mu(A)=\mu\left(T^{-1} A\right)\right)$ preserves the class of measures absolutely continuous wrt Lebesgue measure. By restricting the domain of $T^{*}$ one obtains an operator on $\varrho^{\prime}([0,1], m)$ given by $T^{*}(f)=\left[d T^{*} \mu / d m\right]$, where $d \mu=f d x$ with $f \in \mathcal{L}^{1}$, or equivalently, $\int_{A} T^{*}(f) d x=\int_{T^{-1} A} f d x$ for $f \in \mathcal{L}^{1}$ and every measurable set $A$. This operator is called the Frobenius-Perron (F-P) operator of $T$ and will be denoted by $P_{T}$.

We say $T:[0,1] \rightarrow[0,1]$ is a Lasota-Yorke type map (L-Y type map) if $T$ is piecewise monotone and $C^{2}$, and $T$ is nonsingular. Piecewise monotone and $C^{2}$ means that there is a partition of $[0,1], 0=a_{0}<a_{1}<\cdots<a_{k}=1$, so that for each $i=0,1, \ldots, k-1,\left.T\right|_{\left(a_{i}, a_{i+1}\right)}$ is monotone and extends to a $C^{2}$ map on $\left[a_{i}, a_{i+1}\right]$.

If $T=T_{i}$ (prob. $p_{i}$ ), $i=1, \ldots, n$, is a random map and each $T_{i}$ is nonsingular, we define the F-P operator of $T$ to be $P_{T}(f):=\sum_{i=1}^{n} p_{i} P_{T_{i}}(f)$. Then $P_{T}$ has the following properties:

(i) $P_{T}$ is linear,

(ii) $\left\|P_{T}\right\| \leqslant 1$,

(iii) $P_{T}$ is nonnegative $\left(f \geqslant 0 \Rightarrow P_{T}(f) \geqslant 0\right)$,

(iv) $P_{T}(f)=f \Leftrightarrow d \mu=f d x$ is a $T$-invariant measure,

(v) $P_{T}^{K}(f)=P_{T^{K}}(f)$.

When each $T_{i}$ is a L-Y map we obtain an explicit formula for $P_{T}$ as follows. Let $I_{1}, \ldots, I_{k}$ be a partition of $[0,1]$ into intervals of joint monotonicity of the $T_{i}$ 's. That is, each $T_{i}$ is monotone on $i_{j}$ and extends to a $C^{2}$ function on $\bar{I}_{j}$. Set

$$
H_{i}^{j}=T_{i}\left(I_{j}\right), \quad \psi_{i}^{j}=\left(\left.T_{i}\right|_{I_{j}}\right)^{-1} \text { and } \sigma_{i}^{j}=\left|\frac{d}{d x} \psi_{i}^{j}\right| .
$$

One then computes that

$$
P_{T}(f)(x)=\sum_{i, j} p_{i} f\left(\psi_{i}^{j} x\right) \sigma_{i}^{j}(x) \chi_{H_{i}^{j}}(x),
$$

where $\chi_{I}$ is the characteristic function of the set $I$. 
By (iv), we can prove the existence of an absolutely continuous $T$-invariant measure by showing that $P_{T}$ has an eigenvector for the eigenvalue 1 . This will be accomplished by the use of the mean ergodic theorem [DS]. To show that $P_{T}$ satisfies the hypotheses of the theorem we estimate $V_{0}^{1} P_{T}(f)$ in terms of $\bigvee_{0}^{1} f$, for $f$ a function of bounded variation. The following lemmas are useful for this. The first two appear in $[\mathbf{K}]$.

3. The main theorem. In what follows $B V$ denotes $B V([0,1]), m$ is Lebesgue measure, $\mathcal{L}^{\prime}$ denotes $\mathcal{L}^{\prime}([0,1], m)$ and ' $f \in B V^{\prime}$ ' means $\exists g \in B V$ so that $f=g$ a.e. $(m)$.

Lemma 1. Let $f \in B V([0,1])$ and $\phi \in \mathcal{L}^{\prime}([0,1], m)$. Set $\Phi(x)=\int_{0}^{x} \phi(t) d t$. Then

$$
\left|\int_{0}^{1} f(t) \phi(t) d t\right| \leqslant\left(\underset{x}{\vee} f(t)+\sup _{x}|f(x)|\right)\|\Phi\|_{\infty} .
$$

Lemma 2. Let $f \in B V([0,1])$. Then $\bigvee_{0}^{1} f=\sup _{\phi}\left|\int_{0}^{1} f(t) \phi(t) d t\right|$, where the sup is taken over those $\phi \in \mathcal{L}^{\mathrm{l}}([0,1], m)$ with the properties that (i) $\int_{0}^{1} \phi d t=0$ and (ii) $\sup _{x}\left|\int_{0}^{x} \phi(t) d t\right| \leqslant 1$.

Lemma 3 (Change of Variables). Let $I=[a, b]$ and $f \in B V(I)$. Suppose $T$ : $I \rightarrow J$ is a $C^{1}$ function with $T^{\prime}(x) \neq 0$. Set $\psi=T^{-1}(x)$ and $\sigma=|d \psi(x) / d x|$. Then

$$
\underset{J}{\vee} f(\psi x) \sigma(x) \leqslant\left(\bigvee_{I} f+\operatorname{Sup}_{I}(f)\right)\left(\underset{J}{\vee_{J}}+\underset{J}{\operatorname{Sup} \sigma}\right) .
$$

Proof. Let $\phi$ be as in Lemma 2,

$$
\int_{J}(f \circ \psi) \cdot \sigma \cdot \phi d x=\int_{I} f \cdot z_{\phi} d t, \quad \text { where } z_{\phi}(\psi(x))=\phi(x) .
$$

Hence, by Lemma 1 ,

$$
\left|\int_{J}(f \circ \psi) \cdot \sigma \cdot \phi d x\right|=\left|\int_{I} f \cdot z_{\phi} d t\right| \leqslant\left(\underset{I}{\vee} f+\sup _{I}|f(x)|\right)\left(\sup _{x}\left|\int_{a}^{x} z_{\phi}(t) d t\right|\right) .
$$

But

$$
\begin{aligned}
\int_{a}^{x} z_{\phi} d t & =\int_{a}^{x} \phi(T x) d x=\int_{a}^{x} \phi(T x) \sigma(T x)\left|T_{x}^{\prime}\right| d x \\
& =\int_{T([a, x])} \phi(t) \sigma(t) d t \leqslant\left(\bigvee_{J} \sigma+\sup _{J} \sigma\right)\|\Phi\|_{\infty} .
\end{aligned}
$$

Taking sup over $\phi$ 's as in Lemma 2 completes the proof.

Lemma 4. Let $T, \psi$ and $\sigma$ be as in Lemma 3, but assume that $T$ is $C^{2}$. Then there exist constants $\beta>0$ and $K>0$ so that, for each $f \in B V(I)$,

$$
\vee(f \circ \psi) \sigma \leqslant \beta \bigvee_{I} f+K\|f\|_{1} \text {. }
$$


Proof. First note that

$$
\vee_{l} f+\sup _{l}|f| \leqslant 2 V_{I}(f)+\|f\|_{1} .
$$

Let $J_{1}, \ldots, J_{m}$ be a partition of $J$, and set $I_{i}=\psi\left(J_{i}\right)$. Put $\alpha_{i}=\sup _{J_{t}} \sigma$ and $\alpha=\sup _{J} \sigma$. Then applying Lemma 3 to each $J_{i}$ yields

$$
\begin{aligned}
& \sum_{i=1}^{m} \underset{J_{i}}{\vee} f(\psi(x)) \sigma(x) \leqslant \sum_{i=1}^{m}\left(\underset{I_{i}}{\vee} f f+\int_{I_{i}}|f| d x\right)\left(\alpha_{i}+\underset{J_{i}}{\vee} \sigma\right) \\
& \leqslant 2 \alpha \bigvee_{I} f+2 \sum \underset{I_{\iota}}{\vee}(f) \underset{J_{I}}{\vee} \sigma+\alpha \sum \int_{I_{t}} f|d x| \\
& +\sum V_{J_{i}} \sigma \int_{I_{i}}|f| d x
\end{aligned}
$$

Since $\bigvee_{J_{i}} \sigma=\int_{J_{i}}\left|\sigma^{\prime}\right| d x \leqslant\left(\sup \left|\sigma^{\prime}\right| / \inf \sigma\right) \int_{J_{i}} \sigma d x:=K_{1}$ length $\left(J_{i}\right)$,

$$
\sum \underset{J_{i}}{\vee \sigma} \int_{I_{i}}|f| d x+\alpha \sum \int_{I_{i}}|f| d x \leqslant\left(K_{1}+\alpha\right)\|f\|_{1}:=K\|f\|_{1}
$$

Since $\bigvee_{J_{i}} \sigma \leqslant \sup _{J}\left|\sigma^{\prime}\right|$ length $\left(J_{i}\right)$, we have

$$
2 \sum \underset{I_{t}}{\vee} \underset{J_{t}}{\vee} \boldsymbol{\sigma} \leqslant 2 \sup _{J}\left|\sigma^{\prime}\right|\left(\sum \underset{I_{t}}{\vee} f\right) \cdot \max \left\{\text { length } J_{i}\right\},
$$

and this term may be made arbitrarily small by refining the partition $J_{1}, \ldots, J_{m}$. Combining 1,2 and 3 gives $\bigvee_{J} f(\psi(x)) \sigma(x) \leqslant 2 \alpha \bigvee_{I} f+K\|f\|_{1}$. Note that the $\beta$ of the lemma may be taken as $2 \cdot \sup _{J} \sigma$.

Lemma 4 may be used to provide another proof of Theorem 1 of [LY]. We now prove

THEOREM 1. Let $T(x)=T_{i}(x)$ ( prob. $\left.p_{i}\right), i=1, \ldots, m$, be a random map of $[0,1]$, where each $T_{i}$ is a $L-Y$ type map. If, for all $x \in[0,1]$,

$$
\sum_{i=1}^{m} \frac{p_{i}}{\left|T_{i}^{\prime}(x)\right|} \leqslant \gamma<1
$$

then for all $f \in L^{1}([0,1], m)$ :

(1) The limit

$$
\lim _{n \rightarrow \infty} \frac{1}{n} \sum_{j=0}^{n-1} P_{T}^{j}(f)=f^{*} \text { exists in } \varrho^{1}
$$

(2) $P\left(f^{*}\right)=f^{*}$.

(3) $\vee{ }_{0}^{1} f^{*} \leqslant C\|f\|_{1}$ for some constant $C>0$ which is independent of $f$.

REMARKS. (i) $T_{i}^{\prime}(x)$ may have two values at some points in $[0,1]$, which we denote by $T_{i}^{\prime}(x+)$ and $T_{i}^{\prime}(x-)$. The hypotheses of the theorem require only that

$$
\sum_{i=1}^{m} \frac{p_{i}}{\left|T_{i}^{\prime}(x+)\right|} \leqslant \gamma<1 \text { and } \sum_{i=1}^{m} \frac{p_{i}}{\left|T_{i}^{\prime}(x-)\right|} \leqslant \gamma<1 \text {. }
$$


(ii) Keller $[\mathbf{K}]$ has proved a similar theorem under the stronger hypotheses that, for all $i$ and $x, 1 /\left|T_{i}^{\prime}(x)\right|<1$.

Proof. Our goal is to show that there are constants $0<\alpha<1$ and $K>0$ so that $\bigvee_{0}^{1} P^{N}(f) \leqslant \alpha \bigvee_{0}^{1} f+K\|f\|_{1}$, for some $N \in \mathbf{N}$. This will suffice, as in [LY], to show that $P^{*}=\lim _{n} \frac{1}{n} \sum_{j=0}^{n-1} P^{j}$ exists and is a (nonzero) projection onto the eigenspace $E_{1}$ of eigenvectors of $P_{T}$ with eigenvalue 1 .

Select $N$ so that $\gamma^{N}<1 / 3$, and consider the map $T^{N}$. This is a random map defined by L-Y type functions: $T^{N}(x)=T_{j_{1}} \circ T_{j_{N}, 1} \circ \cdots \circ T_{j_{1}}(x)$ with probability $\Pi_{i=1}^{N} P_{j_{i}}$. The maps defining $T^{N}$ may be indexed by $\{1,2, \ldots, n\}^{N}$. Set $T_{j_{N}} \circ \cdots \circ T_{j_{1}}(x):=T_{w}(x)$ where $w=\left(j_{1}, \ldots, j_{N}\right)$ and $P_{w}=\prod_{i=1}^{N} P_{j_{i}}$. We then have that

$$
\begin{aligned}
& \sum_{n \in\{1,2, \ldots n\}^{N}} \frac{p_{u^{\prime}}}{\left|T_{w^{\prime}}^{\prime}(x)\right|}=\sum_{\overline{u^{\prime}} \in\{1, \ldots, n\}^{N-1}} \sum_{i=1}^{m} \frac{p_{i} p_{\overline{u^{\prime}}}}{\left|T_{i}^{\prime}\left(T_{\overline{u^{\prime}}}(x)\right)\right|\left|T_{w^{\prime}}^{\prime}(x)\right|} \\
& =\sum_{\overline{\bar{w}}} \frac{p_{\overline{w^{-}}}}{\left|T_{\overline{n^{\prime}}}^{\prime}(x)\right|} \sum_{i=1}^{m} \frac{p_{i}}{\left|T_{i}^{\prime}\left(T_{\overline{w^{\prime}}} x\right)\right|} \leqslant \gamma \sum_{\bar{w}} \frac{p_{\overline{w^{\prime}}}}{\left|T_{\bar{w}}^{\prime} x\right|} \leqslant \cdots \leqslant \gamma^{N}<\frac{1}{3} .
\end{aligned}
$$

Thus, to simplify notation we may assume that $\gamma<1 / 3$ and show that $f \in B V \Rightarrow$ $\vee P_{T} f \leqslant \alpha \vee f+K\|f\|_{1}$ for $\alpha \in(0,1)$ and $K>0$.

Recall that $I_{1}, \ldots, I_{l}$ (where $\left.I_{i}=\left(a_{i-1}, a_{i}\right)\right)$ is the partition of $[0,1]$ into maximal intervals of joint monotonicity and $C^{2}$-ness of the maps $\left\{T_{i}\right\}_{i=1}^{m}$. We define

$$
\begin{gathered}
H_{i}^{j}=T_{i}\left(I_{j}\right), \quad i=1, \ldots, m, j=1, \ldots, l, \\
\psi_{i}^{j}=\left(\left.T_{i}\right|_{I_{1}}\right)^{-1} \quad \text { and } \quad \sigma_{i}^{j}=\left|\frac{d}{d x} \psi_{i}^{j}\right| .
\end{gathered}
$$

Let $\mathcal{G}$ be the partition of $[0,1]$ by all the endpoints of the intervals $H_{i}^{j}$, and $\mathcal{K}$ be the partition of $[0,1]$ defined by all inverse images of endpoints of $q$ under all possible $T_{i}$ 's. Each interval of $\mathscr{K}$ is mapped by each $T_{i}$ into exactly one interval of $\mathscr{q}$. Finally, let $\varrho=\left\{L_{k}\right\}$ be a refinement of $\mathscr{K}$ into intervals the maximum length of which will be specified below.

To estimate $\vee{ }_{0}^{1} P_{T}(f)$ we compute as follows: suppose $f \in B V([0,1])$,

$$
\begin{aligned}
V_{0}^{1} \sum_{i=1}^{m} p_{i} \sum_{j=1}^{l} f\left(\psi_{i}^{j}\right) & \sigma_{i}^{j}(x) \chi_{H_{i}^{\prime}} \\
\leqslant & \sum_{i=1}^{m} \sum_{j=1}^{l} p_{i}\left(\left|f\left(a_{j-1}\right)\right| \sigma_{i}^{j}\left(T_{i}\left(a_{j-1}\right)\right)|+| f\left(a_{j}\right) \mid \sigma_{i}^{j}\left(T_{i} a_{j}\right)\right) \\
& +\sum_{i=1}^{m} \sum_{j=1}^{l} p_{i} \vee f\left(\psi_{i}^{j} x\right) \sigma_{i}^{j}(x) .
\end{aligned}
$$

The first sum on the right-hand side of (4) is

$$
\begin{aligned}
\sum_{j=1}^{l}\left(\sum_{i=1}^{m} p_{i} \sigma_{i}^{j}\left(T_{i}\left(a_{j-1}\right)\right)\right)\left|f\left(a_{j-1}\right)\right| & +\left(\sum_{i=1}^{m} p_{i} \sigma_{i}^{j}\left(T_{i}\left(a_{j}\right)\right)\right)\left|f\left(a_{j}\right)\right| \\
& \leqslant \gamma \sum_{j=1}^{l}\left|f\left(a_{j}\right)\right|+\left|f\left(a_{j-1}\right)\right| \leqslant \gamma\left(\bigvee_{I} f+2\|f\|_{1}\right)
\end{aligned}
$$


because

$$
\left|f\left(a_{j-1}\right)\right|+\left|f\left(a_{j}\right)\right| \leqslant I_{I_{l}} f+\underset{I_{l}}{2 \inf }|f| \leqslant \underset{I_{I}}{\vee} f+2 \int|f| d x .
$$

The second sum in (4) we estimate as follows:

$$
\sum_{i=1}^{m} \sum_{j=1}^{l} p_{i} \bigvee f\left(\psi_{i}^{j} x\right) \sigma_{i}^{j}(x) \leqslant \sum_{i=1}^{m} \sum_{j=1}^{l} p_{i} \sum_{\substack{k \text { with } \\ T_{i}\left(I_{K^{K}}\right) \subset H_{i}^{j}}} \underset{T_{i}\left(L_{L_{k}}\right)}{\vee} f\left(\psi_{i}^{j} x\right) \sigma_{i}^{j}(x)
$$

By Lemmas 3 and 4,

$$
\begin{aligned}
& \underset{T_{i}\left(L_{-k}\right)}{\vee} f\left(\psi_{i}^{j} x\right) \sigma_{i}^{j}(x) \leqslant\left(\underset{I_{\cdot k}}{\vee} f+\sup _{L_{k}}(f)\right)\left(\underset{T_{i}\left(L_{L_{k}}\right)}{\vee} \sigma_{i}^{j}+\sup _{T_{i}\left(L_{L_{k}}\right)}\left(\sigma_{i}^{j}\right)\right) \\
& \leqslant 2\left(\sup _{T_{i}\left(I_{K}\right)} \sigma_{i}^{j}\right) \underset{I_{K}}{\vee} f+K_{i k} \int_{L_{L_{K}}}|f| d x \\
& =2 \frac{1}{\inf _{L_{K}}\left|T_{i}^{\prime}(x)\right|} \vee_{I_{K}} f f+K_{i k} \int_{I_{L_{K}}}|f| d x
\end{aligned}
$$

where $K_{i k}=\sup \left|\sigma_{i}{ }^{\prime \prime}\right| / \inf \sigma_{i}{ }^{j}+\sup \sigma_{i}{ }^{j}$. Combining (5) and (6) gives

$$
\begin{aligned}
\sum_{i=1}^{m} \sum_{j=1}^{n} p_{i} \vee f\left(\psi_{i}^{j} x\right) \sigma_{i}^{j}(x) & \leqslant \sum_{k} \sum_{i=1}^{m} \frac{2 p_{i}}{\inf _{L_{K}}\left|T_{i}^{\prime} x\right|} \bigvee_{L_{K}} \vee f+K\|f\|_{1} \\
& \leqslant 2 \gamma^{\prime} \bigvee_{0}^{1} f+K\|f\|_{1}
\end{aligned}
$$

where $\gamma^{\prime}=\max _{k} \sum_{i=1}^{m}\left(p_{i} / \inf _{L_{K}}\left|T_{i}^{\prime}(x)\right|\right)$ and $K=\sum_{i, k} K_{i k}$. Combining (4), (5) and (7) gives

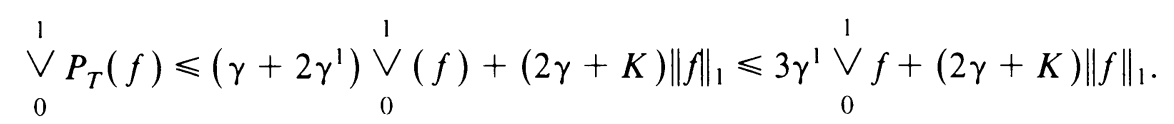

We now show that the partition $\&$ can be chosen so that $3 \gamma^{1}<1$. Select $\delta$ so small that

$$
\sum \frac{p_{i}}{\left|T_{i}^{\prime}(x)\right|+\varepsilon_{i}}<\frac{1}{3} \quad \text { whenever }\left|\varepsilon_{i}\right|<\delta, i=1, \ldots, m .
$$

Then, since $\left.T_{i}\right|_{L_{K}}$ is $C^{2}$, select $\varepsilon$ so that $\left|T_{i}^{\prime}(x)-T_{i}^{\prime}(y)\right|<\delta$ whenever $x$ and $y$ are in

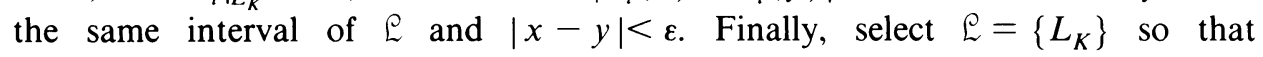
$\max _{k}\left\{\right.$ length $\left.\left(L_{K}\right)\right\}<\varepsilon$. Then

$$
\gamma^{\prime}=\max _{k} \sum_{i=1}^{m} \frac{p_{i}}{\inf _{L_{K}}\left|T_{i}^{\prime} x\right|}<\frac{1}{3}
$$

We have shown that, when $f \in B V[0,1],{ }_{0}^{1} P_{T}(f) \leqslant \alpha \vee_{0}^{1} f+K\|f\|_{1}$ for some $0<\alpha<1$ and $K>0$ independent of $f$. The assertions of the theorem now follow immediately, as in [LY or T]. 
4. An example. In this section we describe a family of random maps which demonstrates that the hypotheses of Theorem 1 are not necessary for the existence of an absolutely continuous invariant measure. The random maps discussed here have the form

$$
T(x)= \begin{cases}f(x) & \text { prob.p } \\ g(x) & \text { prob. } q,\end{cases}
$$

where $f$ is an expanding map $\left(\left|f^{\prime}(x)\right| \geqslant k>1\right)$ and $g$ contracts no faster than $f$ expands $\left(1>\left|g^{\prime}(x)\right| \geqslant 1 / k\right)$. We will impose extra hypotheses and show that when the probability of applying the expanding map is larger than $1 / 2$, there is an absolutely continuous invariant measure. We assume

(1) $g$ is $C^{2}, g(0)=0, g^{\prime \prime}(x) \geqslant 0$ and $\frac{1}{2} \leqslant g^{\prime}<1$ and

(2) $f$ is a L-Y type map with partition $I_{0}, I_{1}, \ldots, I_{n}$; that $f^{\prime}(x) \geqslant 2$ whereever defined, $f(0)=0$ and $f^{\prime \prime}(x) \geqslant 0$ for $x \in I_{0}$, while $f(x) \leqslant x$ for $x \in I_{1}, \ldots, I_{n}$.

The specific case where $f(x)=2 x(\bmod 1)$ and $g(x)=\beta x\left(\frac{1}{2} \leqslant \beta<1\right)$ is an example of a pair of maps which possess properties (1) and (2).

THEOREM 2. Let $T$ be as above, where $f$ and $g$ satisfy properties (1) and (2). If $p>\frac{1}{2}$ then $T$ has an absolutely continuous invariant measure.

Proof. Select $r \in(0,1)$ so that $2^{1-r} \in(1, p / q)$. This can be done since $\lim _{r \rightarrow 1} 2^{1-r}=1$ while $p / q>1$. Changing variables by $y=y(x)=x^{1-r}$ gives a new random map

$$
\tilde{T}(y)= \begin{cases}F(y) & \text { prob. } p, \\ G(y) & \text { prob. } q,\end{cases}
$$

where $F(y)=y(f(x(y)))$ and $G(y)=y(g(x(y)))$. Note that $F$ and $G$ are still L-Y type maps. By the chain rule,

$$
\begin{aligned}
G^{\prime}(y) & =\frac{d y(g(x(y))) / d x}{d y(x(y)) / d x} \cdot \frac{d g}{d x}(x(y)) \geqslant\left(\frac{g(x)}{x}\right)^{-r} \cdot g^{\prime}(x) \\
& \geqslant \frac{g(x)^{1-r}}{x^{1-r}} \geqslant\left(\frac{1}{2}\right)^{1-r}
\end{aligned}
$$

since, by convexity, $g^{\prime}(x) \geqslant g(x) / x$ and $g(x) \geqslant x / 2$. On the interval $y\left(I_{0}\right)$ we compute, as with $G(y)$, that $F^{\prime}(y) \geqslant 2^{1-r}$, while on $y\left(I_{1}\right), \ldots, y\left(I_{n}\right)$ we have

$$
\left|\frac{d F}{d y}\right|=\left|\frac{d y(f(x(y))) / d x}{d y(x(y)) / d x}\right| \cdot\left|\frac{d f}{d x}\right| \geqslant 2 \geqslant 2^{1-r}
$$

since $y^{\prime}$ is decreasing and $f(x) \leqslant x$ on $I_{1}, \ldots, I_{n}$. Hence

$$
\frac{q}{\left|G^{\prime}\right|}+\frac{p}{\left|F^{\prime}\right|} \leqslant q 2^{1-r}+\frac{p}{2^{1-r}}<1
$$

because $q x+p / x<1$ for $x \in(1, p / q)$. Thus $\tilde{T}$ satisfies the hypotheses of Theorem 1 , and so $\tilde{T}$ has an invariant density $\phi$ of bounded variation. From $\phi$ we obtain an invariant density for the random map $T$ by setting $\psi(x)=\phi(y(x)) d y / d x$. Note that $\psi(x)$ is not of bounded variation if $\phi(0)>0$, since $d y / d x=(1-r) x^{-r}$. 


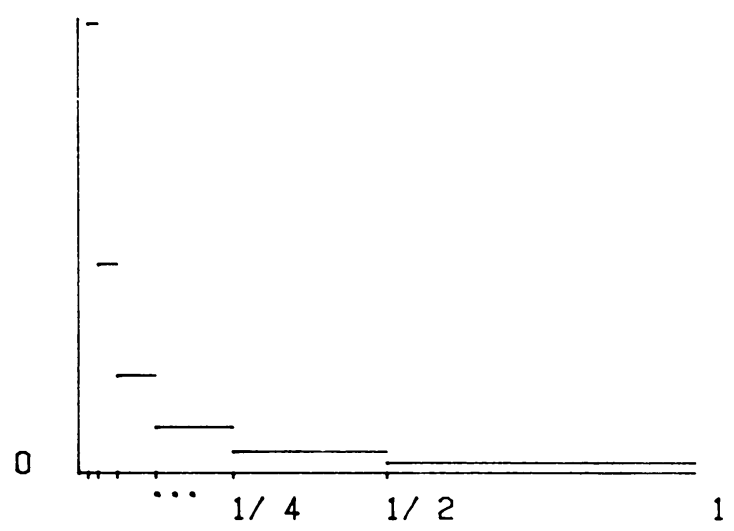

Figure 2. The invariant density of the random map

$$
T(x)=\left\{\begin{array}{ll}
x / 2, & q, \\
2 x \bmod 1, & p,
\end{array} \text { with } 1 / 2<p<2 / 3 .\right.
$$

In the specific case where $f(x)=2 x(\bmod 1)$ and $g(x)=x / 2$ it is possible to construct the density of an absolutely continuous $T$ invariant measure explicitly (Figure 2). In this case we can prove the

Claim. When $p>1 / 2$ the random map $T$ has a unique absolutely continuous invariant measure $\nu_{p}$. The support of $\nu_{p}$ is all of $[0,1]$. The density of the measure, $\left[d \nu_{p} / d m\right]$, is unbounded when $p<2 / 3$. With respect to $\nu_{p}$ the random map $T$ is ergodic in the sense that the associated PSP is ergodic.

Proof. We begin by finding the F-P operator for the random map

$$
T(x)= \begin{cases}T_{1}(x)=2 x \bmod 1, & \text { prob. } p, \\ T_{2}(x)=x / 2, & \text { prob. } q .\end{cases}
$$

Since $T_{2}$ is invertible,

$$
P_{T_{2}}(f)=d\left(T_{2}^{-1}(x)\right) / d x \cdot f\left(T_{2}^{-1}(x)\right) \cdot \chi_{T_{2}[0,1]}=2 f(2 x) \cdot \chi_{[0,1 / 2]} .
$$

The map $T_{1}$ is monotone on 2 intervals on which it has the inverses $x \rightarrow x / 2$ and $x \rightarrow(x+1) / 2$. Hence

$$
P_{T_{1}}(f)=\frac{1}{2} f\left(\frac{x}{2}\right)+\frac{1}{2} f\left(\frac{x+1}{2}\right) .
$$

The F-P operator of $T$ is then

$$
\begin{aligned}
P_{T}(f) & =p P_{T_{1}}(f)+q P_{T_{2}}(f) \\
& =\frac{p}{2}\left(f\left(\frac{x}{2}\right)+f\left(\frac{x+1}{2}\right)\right)+2 q f(2 x) \cdot \chi_{[0,1 / 2]} .
\end{aligned}
$$

Assuming that $P_{T}(f)=f,(9)$ can be rewritten as

$$
f\left(\frac{x}{2}\right)=\frac{2}{p} f(x)-\frac{4 q}{p} \chi_{[0,1 / 2]}(x) f(2 x)-f\left(\frac{x+1}{2}\right) .
$$


Then, assuming $f$ is a positive constant on $(1 / 2,1),(10)$ can be used to define $f$ successively on the intervals $\left(1 / 2^{n+1}, 1 / 2^{n}\right), n=1,2,3, \ldots$ It is immediate that, defined in this way, $f$ is constant on each of these intervals. We now show that $f \geqslant 0$ and that $f \in \mathcal{L}^{\mathrm{l}}([0,1], m)$.

Define $r_{n}=\int_{1}^{1 / 2^{n+1}} f(x) d x$, and $a_{n}=\left.f\right|_{\left(1 / 2^{n} \cdot 1.1 / 2^{n}\right)}$. Integrating (10) and changing variables we have

$$
\begin{gathered}
r_{n}=\frac{1}{p} r_{n-1}-\frac{q}{p} r_{n-2}-\frac{1}{2^{n+1}} r_{0}, \quad n \geqslant 2, \\
a_{n}=\frac{2}{p} a_{n-1}-\frac{4 q}{p} a_{n-2}-a_{0}, \quad n \geqslant 2 \text { and } 2^{n+1} r_{n}=a_{n} .
\end{gathered}
$$

Then

$$
\left(\begin{array}{c}
r_{n+1} \\
r_{n}
\end{array}\right)=A\left(\begin{array}{c}
r_{n} \\
r_{n-1}
\end{array}\right)-\frac{1}{2^{n+1}}\left(\begin{array}{c}
r_{0} \\
0
\end{array}\right), \quad \text { where } A=\left(\begin{array}{cc}
1 / p, & -q / p \\
1, & 0
\end{array}\right)
$$

so that

$$
\begin{aligned}
\left(\begin{array}{c}
r_{n+1} \\
r_{n}
\end{array}\right) & =A^{n+1}\left(\begin{array}{c}
r_{0} \\
0
\end{array}\right)-\sum_{j=0}^{n} A^{n-j} \frac{1}{2^{j+1}}\left(\begin{array}{c}
r_{0} \\
0
\end{array}\right) \\
& =\left(A^{n+1}-\frac{1}{2^{n+1}} \sum_{j=0}^{n} 2^{j} A^{j}\right)\left(\begin{array}{c}
r_{0} \\
0
\end{array}\right) .
\end{aligned}
$$

The eigenvalues (eigenvectors) of $A$ are $\lambda_{1}=1, e_{1}=(1,1)$ and $\lambda_{2}=q / p, e_{2}=$ $(1, p / q)$. With respect to the basis $e_{1}, e_{2}$ the matrix in (11) may be rewritten as

$$
A^{n+1}-\sum_{j=0}^{n} A^{n-j} \cdot \frac{1}{2^{j+1}}=\left(\begin{array}{cc}
\frac{1}{2}^{n+1} & 0 \\
0 & \left(\frac{q}{p}\right)^{n+1}-\frac{\left(1^{n+1} / 2\right)-(q / p)^{n+1}}{(p-2 q) / p}
\end{array} \mid .\right.
$$

Hence, $r_{n+1} / r_{n}=\left(a b-q c_{n}\right) /\left(a b-p c_{n}\right)$ where

$$
a=p /(p-q), \quad b=1 /(p-q)(p-2 q)
$$

and

$$
c_{n}=2^{n+1}\left(2(q / p)^{n}\left(1-p^{n-1}\right)-p\right) .
$$

When $2 q / p>1$, so that $p<2 / 3$, we have that $c_{n} \rightarrow \infty$ as $n \rightarrow \infty$ and $\lim _{n}\left(r_{n+1} / r_{n}\right)$ $=q / p$. But $q / p<1$ for $p>1 / 2$, and so $\int_{0}^{1} f d x=\sum_{i=0}^{\infty} r_{i}<\infty$ converges by the ratio test. For $1 / 2<p<2 / 3$ we also have that

$$
\frac{a_{n+1}}{a_{n}}=\frac{2^{n+1} r_{n+1}}{2^{n} r_{n}} \sim \frac{2 q}{p}>1,
$$

so that $\left\{a_{n}\right\}$ is increasing and unbounded. Thus, when normalized, $f$ is the density of an invariant probability measure $\nu_{p}$. The assertion concerning the uniqueness of $\nu_{p}$ and the ergodicity of $T$ follows from the fact that $\nu_{p}$ is equivalent to Lebesgue measure and Corollary 7 of the next section. 
5. Ergodic components. In this section we continue to assume that $T(x)=T_{i}(x)$ (prob. $p_{i}$ ), $i=1, \ldots, n$, is a random map of $[0,1]$ specified by L-Y type maps, and that $T$ satisfies the hypotheses of Theorem 1. $R$ denotes the PSP associated with $T$ (see $\S 2$ ) and $\mu_{f}$ denotes the measure with $d \mu_{f} / d m=f$.

The proof of Theorem 1 shows that some power of $P_{T}$ is quasicompact as an operator on $B V$. This implies (see $[\mathbf{K}]$ ) that the eigenspace $E_{1}$ corresponding to the eigenvalue 1 is a finite-dimensional vector sublattice of $B V \subset \mathcal{L}^{\mathrm{l}}$. In particular, $E_{1}$ has a basis of nonnegative functions $v_{1}(x), \ldots, v_{k}(x)$ with $\min \left(v_{i}, v_{j}\right)=0$ when $i \neq j$. Set $A_{i}=\operatorname{support}\left(v_{i}\right)=\left\{x: v_{i}(x)>0\right\}$. Since $v_{i} \in B V$, each $A_{i}$ differs from a union of intervals by a set of zero Lebesgue measure.

In this section it will be shown that the PSP $R$ is ergodic with respect to each measure $\mu_{p} \times \mu_{v_{i}}$. The projection

$$
P^{*}\left(\chi_{[0,1]}\right)=\lim _{n} \frac{1}{n} \sum_{j=0}^{n-1} P^{j}\left(\chi_{[0,1]}\right):=1^{*}
$$

is nonzero onto each basis vector $v_{i}(x)$. Hence, the number of ergodic components of $R$ wrt $\mu_{p} \times \mu_{1^{*}}$ is the maximum possible for any product measure the second factor of which is absolutely continuous wrt Lebesgue measure. The sets $A_{i}$ are invariant under $T$ in the sense of the following

Lemma 5. $m\left(A_{i} \triangle \cup_{j=1}^{n} T_{j}\left(A_{i}\right)\right)=0$.

Proof. Set $S=A_{i} \backslash \cup_{j=1}^{n} T_{i}\left(A_{i}\right)$. If $m(S)>0$, then $\mu_{v_{i}}(S)>0$ since $S \subset A_{i}$. But $T^{-1}(S)=\cup_{j=1}^{n} T_{i}^{-1}(S) \subset A_{i}^{c}$ so $\mu_{v_{i}}\left(T^{-1} S\right)=0$, which contradicts the fact $\mu_{v_{\imath}}$ is $T$-invariant. Similarly, suppose $S=\cup_{j=1}^{n} T_{j}\left(A_{i}\right) \backslash A_{i}$ has $m(S)>0$. Then $\mu_{v_{i}}(S)=0$ because $S \subset A_{i}^{c}$ while $\mu_{v_{i}}\left(T^{-1} S\right)>0$ because each $T_{i}$ is nonsingular with respect to $m$ and hence $m\left(T^{-1} S \cap A_{i}\right)>0$. Again, this contradicts the assumption that $\mu_{v_{1}}$ is $T$ invariant.

The significance of the sets $A_{i}$ is seen by the following characterization of the invariant sets of the PSP $R$.

Proposition 6. Suppose that $\mu=\mu_{p} \times \nu$ is $R$-invariant and that $A \subset \Omega \times I$ is an invariant set of positive $\mu$ measure $(R(A) \subset A$ and $\mu(A)>0)$. Then there is a set $B \subset[0,1]$ so that $\mu(A \triangle(\Omega \times B))=0$, and $B$ is T-invariant in the sense that $\nu\left(B \triangle \cup_{j=1}^{n} T_{j}(B)\right)=0$.

Proof. First note that if a measurable set $U \subset \Omega$ has $\mu_{p}(U)>0$, then $\lim _{n} \mu_{p}\left(\sigma^{n}(U)\right)=1$. Suppose that $A$ is not of the form $\Omega \times B$. Then $\exists S \subset[0,1]$ with $\nu(S)>0$ so that for $\nu$ almost every $x \in S$ we have $0<\mu_{p}\left(A_{x}\right)<1-\varepsilon$, where $A_{x}=\{\omega \in \Omega:(\omega, x) \in A\}$ is the $x$-section and $\varepsilon$ is some positive number. Define a sequence of measurable functions by $f_{n}(x)=\mu_{p}\left(\sigma^{n}\left(A_{x}\right)\right)$. Then $f_{n} \rightarrow 1$ pointwise almost everywhere on $S$, and there is a set $S^{\prime} \subset S$ with $\nu\left(S^{\prime}\right)>0$ so that $f_{n}(x) \rightarrow 1$ uniformly on $S^{\prime}$. Select $N$ so that $n \geqslant N$ and $x \in S^{\prime}$ implies $f_{n}(x) \geqslant 1-\varepsilon / 2$. Poincare recurrence guarantees that almost every point of $A \cap\left(\Omega \times S^{\prime}\right)$ returns to it 
under the iterates $R^{N+j}, j=0,1,2, \ldots$, and consequently $\nu$-almost every $x \in S^{\prime}$ has $\mu_{p}\left(A_{x}\right)>1-\varepsilon / 2$, contradicting the definition of the set $S$. We have shown that $A=\Omega \times B$ (except for a set of zero $\mu_{p} \times \nu$ measure). By the invariance of the set $A$, it is immediate that $\cup_{j=1}^{n} T_{j}(B) \subset B$. Since $R(A) \subset A$, we have $\mu\left(R^{-1}(A) \backslash A\right)=0$. This implies that $\mu(A \backslash T A)=0$, and hence $\nu\left(B \backslash \cup_{j=1}^{n} T_{j} B\right)=0$.

The assumption that $v_{1}(x), \ldots, v_{k}(x)$ is a basis for $E_{1}$ implies that no $A_{i}$ has a proper subset $\left(S \subset A, 0<\mu_{v_{1}}(S)<\mu_{v_{1}}(A)\right.$ ) which is invariant in the sense of Lemma 5. This fact, together with the previous proposition establishes a 1-1 correspondence between the sets $A_{i}$ and the ergodic components of $R$ wrt $\mu_{p} \times \mu_{1^{*}}$.

We now have enough information to describe "almost every" trajectory of the random map $T$. Set $A_{j}^{-\infty}=\left\{(w, x): R^{k}(w, x) \in \Omega \times A_{j}\right.$ for some $\left.k \geqslant 0\right\}=$ $\cup_{k=0}^{\infty} R^{-k}\left(\Omega \times A_{j}\right)$. If $(w, x) \in A_{j}^{-\infty}$, the distribution of the sequence $\left\{x_{n}\right\}$ (where $\left.x_{n}=T_{w_{n}} \circ \cdots \circ T_{w_{0}}(x)\right)$ is given by $\mu_{v}$. Since, $\cup_{j=1}^{k} A_{j}^{-\infty}=\Omega \times I$, up to sets of measure zero wrt $\mu_{p} \times m$, this is a "complete" description of the ways in which trajectories of the random map $T$ can be distributed. We now assume that one of the maps specifying $T$, say $T_{1}$, is an expanding map $\left(\left|T_{1}^{\prime}(x)\right|>1\right.$ wherever defined). For such a map $\mathrm{Li}$ and Yorke [LiY] have shown that (with respect to any absolutely continuous $T_{1}$-invariant measure) there are a finite number of ergodic components. They also show that each component must contain at least one point of discontinuity of the function $d T_{1}(x) / d x$. This result gives an easy method for determining an upper bound for the number of ergodic components of an expanding L-Y type map. Because of the fact that the ergodic components of the PSP $R$ associated with a random map $T$ are products, we may apply the Li-Yorke result as follows.

Corollary 7. Suppose $T_{1}$ is an expanding map. Let $C_{1}, \ldots, C_{k}$ be the ergodic components of $T_{1}$ wrt the measure $\mu\left(d \mu=P_{T_{1}}^{*}\left(\chi_{[0,1]}\right) d x\right)$. Define $C_{i} \sim C_{j}$ if $m\left(T_{i_{1}} \circ T_{i_{1-1}} \circ \ldots \circ T_{i_{1}}\left(C_{i}\right) \cap C_{j}\right)>0$, for some $\left(i_{1}, \ldots, i_{1}\right)$. Then $\sim$ is an equivalence relation, and the number of ergodic components of $R$ wrt $\mu_{p} \times \mu_{1^{*}}$ is the number of $\sim$ equivalence classes. In particular, the number of ergodic components of $R$ is bounded by the number of discontinuities of $d T_{1} / d x$.

Proof. Under the iterates of $T_{1}$ almost every $(m)$ point is mapped into $C=\cup C_{i}$. This implies, because $A_{i}$ is invariant in the sense of Lemma 5, that each set $\Omega \times A_{i}$ contains at least one set of the form $\Omega \times C_{j}$. Thus each $C_{j} \subset \operatorname{support}\left(1^{*}\right)$ and $\mu_{1_{*}}\left(C_{j}\right)>0$. To show that $\sim$ is an equivalence relation: $C_{i} \sim C_{i}$ since $T_{1}\left(C_{i}\right) \subset C_{i}$. It follows from the definition that $\sim$ is transitive. The fact that $\sim$ is symmetric follows from the Poincaré recurrence theorem, since $\mu_{1^{*}}\left(C_{i}\right)>0$. By Proposition $6, C_{i} \sim C_{j}$ iff $\Omega \times C_{i}$ and $\Omega \times C_{j}$ are in the same ergodic component of $R$.

\section{REFERENCES}

[AR] L. M. Abramov and V. A. Rohlin, The entropy of a skew product of measure-preserving transformations, Amer. Math. Soc. Transl. (2) 48 (1965), 255-265.

[DS] N. Dunford and J. T. Schwartz, Linear operators. I, Pure and Appl. Math., Vol. 7, Interscience, New York, 1958.

[K] G. Keller, Stochastic stability in some chaotic dynamical systems, preprint. 
[LiY] T.-Y. Li and J. A. Yorke, Ergodic transformations from an interval into itself, Trans. Amer. Math. Soc. 235 (1978), 183-192.

[LY] A. Lasota and J. A. Yorke, On the existence of invariant measures for piecewise monotonic transformations, Trans. Amer. Math. Soc. 186 (1973), 481-488.

[T] C. Ionescu-Tulcea and G. Marinescu, Théorie ergodique pour des classes d'opérations noncompletement continues, Ann. of Math. (2) 52 (1950), 140-147.

Department of Mathematics, University of Maryland, College Park, Maryland 20742

Current address: Institute for Mathematics and its Applications, University of Minnesota, Minneapolis, Minnesota 55455 